Pathologe 2010 · 31:89-90

DOI 10.1007/s00292-009-1251-z

Online publiziert: 9. Januar 2010

(c) Springer-Verlag 2010
T. Mentzel ${ }^{1} \cdot$ C. Kuhnen ${ }^{2}$ I. Leuschner ${ }^{3}$

${ }^{1}$ Dermatopathologische Gemeinschaftspraxis, Friedrichshafen

${ }^{2}$ Institut für Pathologie am Clemenshospital, Medical Center, Münster

${ }^{3}$ Institut für Pathologie, Universität Kiel

\title{
Mesenchymale Tumoren der Haut und des Weichgewebes
}

\author{
Neue Entitäten und Konzepte
}

Mesenchymale Tumoren der Haut und des Weichgewebes sind bekanntermaßen im Vergleich zu epithelialen, melanozytären und lymphatischen Neoplasien sehr selten und zudem durch eine außerordentliche klinisch-pathologische Heterogenität charakterisiert. In den letzten Jahren wurden bei einer Reihe benigner und maligner mesenchymaler Tumoren distinkte und reproduzierbare genetische Veränderungen nachgewiesen, die sowohl eine wichtige diagnostische als auch zunehmend eine prognostisch-therapeutische Bedeutung besitzen. Diese Charakterisierung auf genetischer Ebene und die Bildung von Referenzzentren, die eine immer größere Anzahl dieser seltenen Tumoren untersuchen können, haben nicht nur zur Beschreibung und Charakterisierung einer Reihe neuer klinisch-pathologischer Entitäten geführt, sondern auch die Klassifizierung mesenchymaler Tumoren entscheidend modifiziert.

So werden neben benignen und malignen mesenchymalen Neoplasien in der derzeit gültigen WHO-Klassifikation zwei Kategorien von früher als sog. „Borderline-Tumoren “ bezeichneten Neoplasien unterschieden. Intermediär lokal aggressive, jedoch nichtmetastasierende Weichgewebstumoren, wie z. B. die Desmoidfibromatosen, sind durch eine erhöhte Lokalrezidivrate sowie ein invasives und lokal destruierendes Wachstum charakterisiert, besitzen aber kein Metastasierungs- potenzial. Dagegen können sich bei intermediär lokal aggressiven und selten metastasierenden Tumoren wie z. B. dem plexiformen fibrohistiozytischen Tumor oder dem angiomatoiden fibrösen Histiozytom in seltenen Fällen ( $<2 \%$ der Fälle) Metastasen entwickeln.

Die Beschreibung und Charakterisierung so genannter neuer Entitäten ist dann sinnvoll, wenn es sich um eine klinisch-pathologische Entität mit einer distinkten und reproduzierbaren Morphologie und teilweise auch Genetik mit klinisch-pathologischen sowie auch therapeutischen Unterschieden zu bereits bestehenden Entitäten handelt oder wenn differenzialdiagnostisch bedeutsame Befunde vorliegen. Dies trifft insbesondere für biologisch benigne Tumoren zu, die mit malignen Neoplasien verwechselt werden können (z. B. chondroides Lipom) oder für biologisch maligne Neoplasien, die benigne Tumoren imitieren (z. B. niedrig malignes fibromyxoides Sarkom).

Des Weiteren wurden einige in der Vergangenheit als separate Entitäten aufgelistete Tumoren aufgrund vergleichbarer Klinik und Genetik zusammengefasst (z. B. Spindelzelllipom und pleomorphes Lipom; atypisches Lipom und gut differenziertes Liposarkom zum atypischen lipomatösen Tumor; niedrig malignes fibromyxoides Sarkom und hyalinisierender spindelzelliger Tumor mit Rie- senrosetten; inflammatorischer myofibroblastischer Tumor und inflammatorisches Fibrosarkom). Schließlich hat es sich in den letzten Jahren herausgestellt, dass das "maligne fibröse Histiozytom“ und das „Hämangioperizytom“ "keine distinkten klinisch-pathologischen Entitäten repräsentieren, sondern jeweils lediglich ein Wuchsmuster darstellen, das von Neoplasien unterschiedlicher Differenzierungsrichtungen verwirklicht wird.

Nachdem in vorangegangenen Ausgaben von „Der Pathologe“ bereits auf die Entwicklung des Konzepts atypischer lipomatöser Tumoren [1] sowie des „malignen fibrösen Histiozytoms “ [2] eingegangen wurde, sollen im vorliegenden Heft mit der Lipofibromatose, dem spindelzelligen Rhabdomyosarkom des Erwachsenenalters und dem Gardner-Fibrom einige der in den letzten Jahren charakterisierten mesenchymalen Tumoren vorgestellt werden. Des Weiteren wird die Entwicklung des Konzepts des inflammatorischen myofibroblastischen Tumors, der gastrointestinalen Stromatumoren, des „Hämangioperizytoms“, des inflammatorischen fibroiden Polypen des Gastrointestinaltrakts und der malignen Transformation benigner Lipome besprochen, und es werden ein mögliches Entwicklungskonzept des desmoplastischen kleinund rundzelligen Tumors sowie ein Konzept der differenzialdiagnostisch bedeut- 
samen Formvarianten des epitheloiden Sarkoms dargestellt.

Bei all der teilweise verwirrenden Vielfalt mesenchymaler Tumoren der Haut und des Weichgewebes ist die sinnvolle Integration molekularpathologischer Befunde in die Diagnose und Beurteilung der Biologie dieser Geschwülste von großer Bedeutung, hat teilweise zu einer Vereinfachung der Klassifikation geführt und wird bei einigen Entitäten sicherlich auch in Zukunft eine zunehmende therapeutische Konsequenz haben. Andererseits - und dies macht mesenchymale Neoplasien so interessant und reizvoll - ist die genaue morphologische Analyse dieser Neoplasien und die Korrelation mit klinischen und genetischen Befunden sehr wichtig und unterstreicht die Rolle des Pathologen bei der Diagnosefindung und bei zu treffenden Therapieentscheidungen. Aufgrund der Seltenheit dieser Neoplasien empfiehlt sich insbesondere bei problematischen Fällen die Hinzuziehung eines Referenzzentrums.
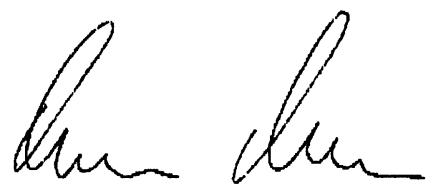

T. Mentzel
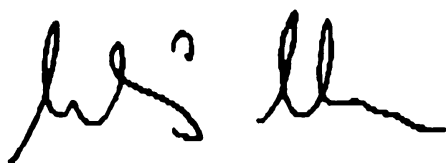

C. Kuhnen

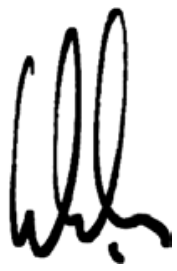

I. Leuschner

\section{Korrespondenzadresse}

\section{Prof. Dr. T. Mentzel}

Dermatopathologische Gemeinschaftspraxis Siemensstr. 6/1, 88048 Friedrichshafen

mentzel@dermpath.de

\section{Literatur}

1. Mentzel T (2000) Lipomatöse Tumoren der Haut und des Weichgewebes. Neue Entitäten und Konzepte. Pathologe 21:441-448

2. Meister P (2005) Das maligne fibröse Histiozytom. Undifferenziertes pleomorphes Sarkom oder pleomorphes Fibrosarkom? Pathologe 26:127-133

\section{AJCC Cancer Staging}

Zeitgemäße Klassifikation

von Tumorerkrankungen

Eine korrekte Klassifikation von Tumorerkrankungen in verschiedene Stadien aufgrund international gültiger Regeln erlaubt dem behandelnden Arzt prognostische Aussagen zu treffen und angemessene Behandlungstherapien zu wählen. Untersuchungs- und Forschungsergebnisse von Krebserkrankungen sind somit auch auf internationaler Ebene besser miteinander vergleichbar. Das AJCC Cancer Staging Manual und das dazugehörige Handbook, beide herausgegeben vom American Joint Committee on Cancer, stellen Standardwerke bezüglich der Klassifikation von Neoplasien hinsichtlich ihrer anatomischen Ausbreitung dar. In der 7. Auflage geben beide Werke, neben den derzeit verfügbaren Informationen über das Klassifikationssystem, auch den aktuellen Wissensstand in Bezug auf die Ätiologie und Pathologie von Krebserkrankungen wieder. Das kompakte Handbuch beinhaltet den kompletten Text des Manuals, allerdings im handlicheren Format. Das AJCC Cancer Staging Manual stellt zusätzlich zum Handbuch am Ende jedes einzelnen Kapitels die Stadienformen der jeweils vorgestellten Krebserkrankungen dar.

Die beiden Ausgaben beinhalten u.a. folgende Themen:

- Prinzipien und Regeln der TNMKlassifikation

- Zweck der TNM-Klassifikation

- Übersicht zu den Neuheiten in der TNMKlassifikation

- Krebsüberlebensraten

Bestellen Sie das

AJCC Cancer Staging Manual

(7. Aufl., 2010, 646 S., 130 Abb., mit CD-ROM, ISBN: 978-0-387-88440-0) zum Preis von 49,95 EUR bzw. das AJCC Cancer Staging Handbook (7. Aufl., 2010, 730 S., 130 Abb., ISBN: 978-0-387-88442-4) zum Preis von 34,95 EUR auf

www.springer.com 